\title{
Very high resolution crop surface models (CSMs) from UAV-based stereo images for rice growth monitoring In Northeast China
}

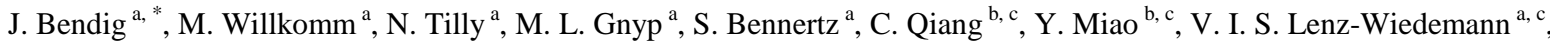 \\ G. Bareth ${ }^{\mathrm{a}, \mathrm{c}}$

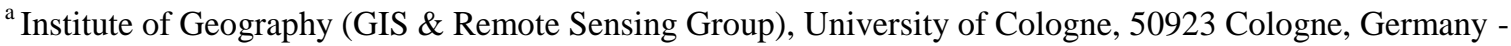 \\ (juliane.bendig, m.willkomm, nora.tilly, mgnyp1, s.bennertz, victoria.lenz, g.bareth)@uni-koeln.de \\ ${ }^{\mathrm{b}}$ College of Resources and Environmental Sciences, China Agricultural University, Beijing, 100094, China - \\ (qiangcao, ymiao)@cau.edu.cn \\ ${ }^{c}$ ICASD - International Center for Agro-Informatics and Sustainable Development (www.icasd.org)
}

KEY WORDS: Agriculture, Biomass, DEM, Multi-temporal data, Plant height, Rice, UAV

\begin{abstract}
:
Unmanned aerial vehicles (UAVs) became popular platforms for the collection of remotely sensed geodata in the last years (Hardin \& Jensen 2011). Various applications in numerous fields of research like archaeology (Hendrickx et al., 2011), forestry or geomorphology evolved (Martinsanz, 2012). This contribution deals with the generation of multi-temporal crop surface models (CSMs) with very high resolution by means of low-cost equipment. The concept of the generation of multi-temporal CSMs using Terrestrial Laserscanning (TLS) has already been introduced by Hoffmeister et al. (2010). For this study, data acquisition was performed with a low-cost and low-weight Mini-UAV $(<5 \mathrm{~kg})$. UAVs in general and especially smaller ones, like the system presented here, close a gap in small scale remote sensing (Berni et al., 2009; Watts et al., 2012). In precision agriculture frequent remote sensing on such scales during the vegetation period provides important spatial information on the crop status. Crop growth variability can be detected by comparison of the CSMs in different phenological stages. Here, the focus is on the detection of this variability and its dependency on cultivar and plant treatment. The method has been tested for data acquired on a barley experiment field in Germany. In this contribution, it is applied to a different crop in a different environment. The study area is an experiment field for rice in Northeast China (Sanjiang Plain). Three replications of the cultivars Kongyu131 and Longjing21 were planted in plots that were treated with different amounts of N-fertilizer. In July 2012 three UAV-campaigns were carried out. Establishment of ground control points (GCPs) allowed for ground truth. Additionally, further destructive and non-destructive field data were collected. The UAV-system is an MK-Okto by Hisystems (www.mikrokopter.de) which was equipped with the high resolution Panasonic Lumix GF3 12 megapixel consumer camera. The self-built and self-maintained system has a payload of up to $1 \mathrm{~kg}$ and an average flight time of 15 minutes. The maximum speed is around $30 \mathrm{~km} / \mathrm{h}$ and the system can be operated up to a wind speed of less than $19 \mathrm{~km} / \mathrm{h}$ (Beaufort scale number 3 for wind speed). Using a suitable flight plan stereo images can be captured. For this study, a flying height of $50 \mathrm{~m}$ and a $44 \%$ side and $90 \%$ forward overlap was chosen. The images are processed into CSMs under the use of the Structure from Motion (SfM)-based software Agisoft Photoscan 0.9.0. The resulting models have a resolution of $0.02 \mathrm{~m}$ and an average number of about 12 million points. Further data processing in Esri ArcGIS allows for quantitative comparison of the plant heights. The multi-temporal datasets are analysed on a plot size basis. The results can be compared to and combined with the additional field data. Detecting plant height with non-invasive measurement techniques enables analysis of its correlation to biomass and other crop parameters (Hansen \& Schjoerring, 2003; Thenkabail et al., 2000) measured in the field. The method presented here can therefore be a valuable addition for the recognition of such correlations.
\end{abstract}

\section{INTRODUCTION}

Surveying crop growth during phenological stages is an important component of precision agriculture (Hansen \& Schjoerring, 2003; Thenkabail et al., 2000). Remote sensing has great potential of contributing data for such kind of investigations in the field of precision agriculture (Mulla, 2012). In Northeast China, rice production is an important economic factor and contributes to ensuring the food supply for the local population (Miao et al., 2011; Peng et al., 2006). A way to monitor plant growth is the idea of generating multitemporal crop surface models (CSMs) to allow for comparison of different phenological stages (Hoffmeister et al., 2010;
Bendig et al., 2013). For each date of data acquisition a model of the crop surface is generated from highly dense point clouds. The plant growth is obtained by comparison of the surface models for each date. In this study the point clouds were generated from very high resolution stereo images captured by a UAV-system.

The data collection using such a mobile, self-maintainable device offers big advantages in this remote region of the world. In addition, the well-managed, small-sized experiment field provides an ideal opportunity to validate the method of CSM generation by a UAV. author. 
The aim of this contribution is to apply the method developed in Germany to data collected under different conditions such as an irrigated field crop.

\section{DATA AQUISITION}

\subsection{Study Area and Dataset}

The study area is Keyansuo experiment field in China's Sanjiang Plain the northernmost rice growing region in China (Figure 1). Two cultivars of rice seedlings were transplanted in May of 2012. Ground water was used for irrigation during the growing season. Harvesting of the crops took place at the end of September. The experiment consisted of 54 small plots with a size of $7 \times 8 \mathrm{~m}$, where three replications of the rice varieties Kongyu131 and Longjing21 were planted. Different amounts of $\mathrm{N}$-fertilizer $\left(0-160 \mathrm{~kg} \mathrm{ha}^{-1}\right)$ were applied (Figure 2). The total size of the field is 0.39 ha.

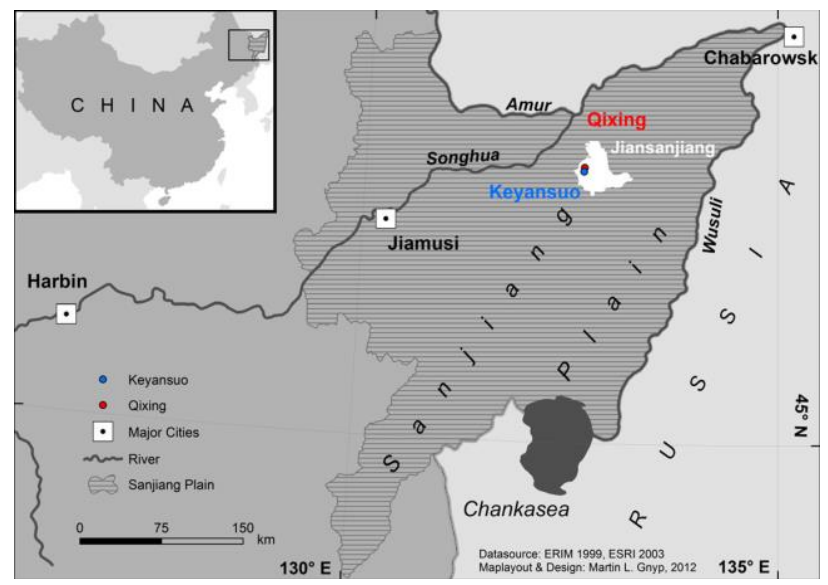

Figure 1. Location of the experiment fields Qixing and Keyansuo, Jiansanjiang Branch Bureau, Heilongjiang Bureau of Agricultural Reclamation, Heilongjiang province, China. (Kang et al., 2013).

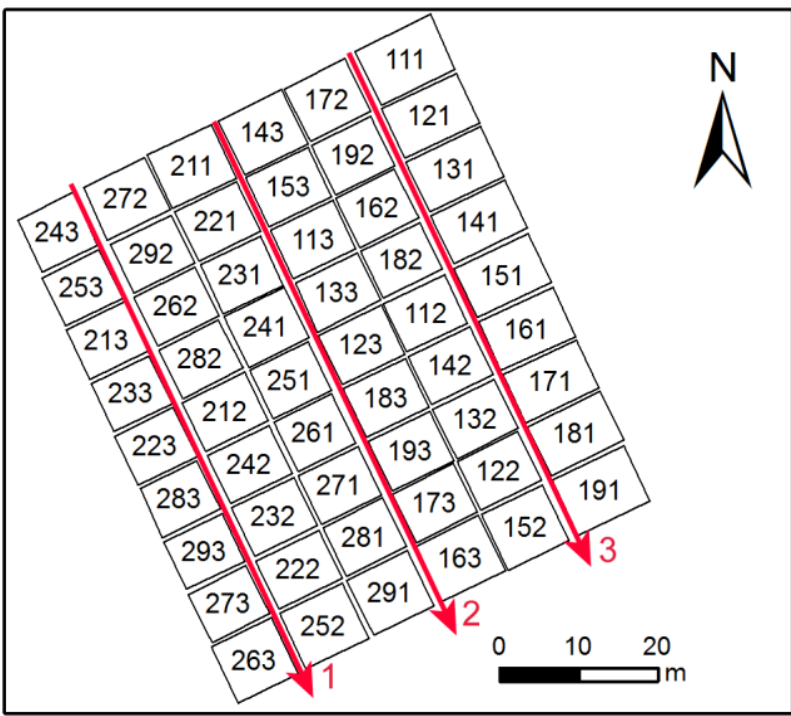

Figure 2. Experiment field in Keyansuo, plot numbering: $1^{\text {st }}$ no.: cultivar ( $1=$ Kongyu $131,2=$ Longjing21), $2^{\text {nd }}$ no.: treatment $(1=0,2=70,3=100,4=130,5=160$ $\mathrm{kg} \mathrm{ha}^{-1}, 6-9=$ other$), 3^{\text {rd }}$ no.: replication, red arrows: flight strips $1-3$.
In July 2012 three UAV-campaigns (04./09./17.09.12) were carried out. 30 ground control points (GCPs) were distributed evenly across the field to facilitate ground truth.

Additional destructive sampling of biomass and non-destructive measurements of plant height, hyperspectral data and 3D point clouds using a terrestrial laser scanner were carried out.

\subsection{Platform}

An MK-Okto by hisystems (Hisystems $\mathrm{GmbH}, 2013$ ) is chosen as sensor platform. The UAV-system was self-built at the study site in 2011. Thus maintenance could take place on site as well. The frame consists of aluminum and fiberglass reinforced plastics (Figure 3). The eight engines are equipped with high performance propellers. The electronics consist of an ARMprocessor equipped mainboard and a navigation mainboard with gyroscopes, a pressure sensor and a module compass module (Bendig et al., 2012). Lithium polymer batteries (up to $6600 \mathrm{mAh}$ capacity) are used for the power supply. A $2.4 \mathrm{GHz}$ transmitter remote control (RC) is used for stirring and camera triggering. The system can carry up to $1 \mathrm{~kg}$ of payload, has an average flight time of approx. 15 minutes and costs approx. $3000 €$ including RC. Operation is possible up to a wind speed of $19 \mathrm{~km} \mathrm{~h}^{-1}$ (Beaufort scale number 3 for wind speed).

\subsection{Sensor}

The Panasonic Lumix DMC GF3 combined with a Lumix G $20 \mathrm{~mm}$ (F1.7 ASPH) fixed lens was chosen as RGB sensor. The camera has a weight of $400 \mathrm{~g}$. The sensor resolution of $4016 \times 3016$ (12 million) pixel (Panasonic, 2013) enables capturing very high resolution images of $0.01 \mathrm{~m}$ at a $50 \mathrm{~m}$ object distance. The Field of View (FOV) is $48.5^{\circ}$ horizontal and $33.4^{\circ}$ vertical, resulting in an image size of $45 \times 30 \mathrm{~m}$ at a $50 \mathrm{~m}$ object distance.

Prior to each flight aperture and exposure time are adjusted and fixed manually according to the light conditions. The camera holder is individually adapted and features a mechanical trigger which is controlled by the remote control of the UAV-system.

\subsection{Measurement}

Prior to the flight 30 wooden poles were installed on the dikes between the plots with a uniform distribution over the study area. $0.3 \times 0.3 \mathrm{~m}$ highly visible targets were attached to the poles and served as GCPs.

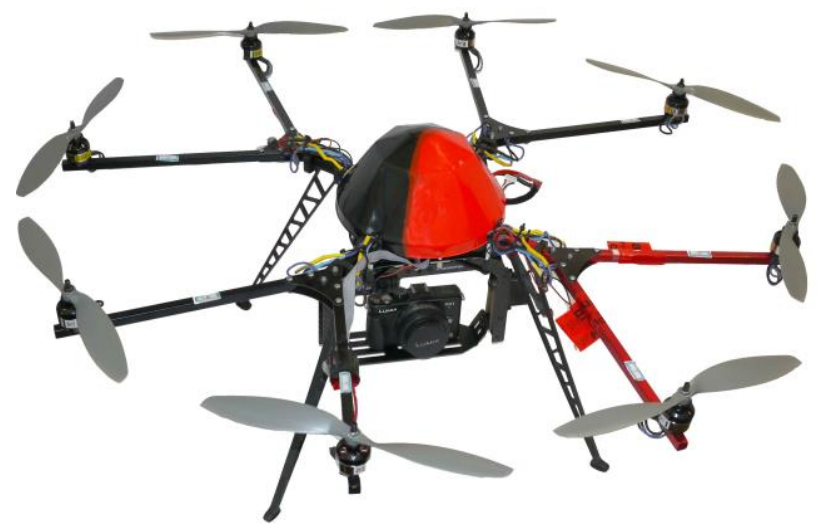

Figure 3. MK-Okto by Hisystems GmbH mounted with Lumix DMC GF3 consumer camera. 


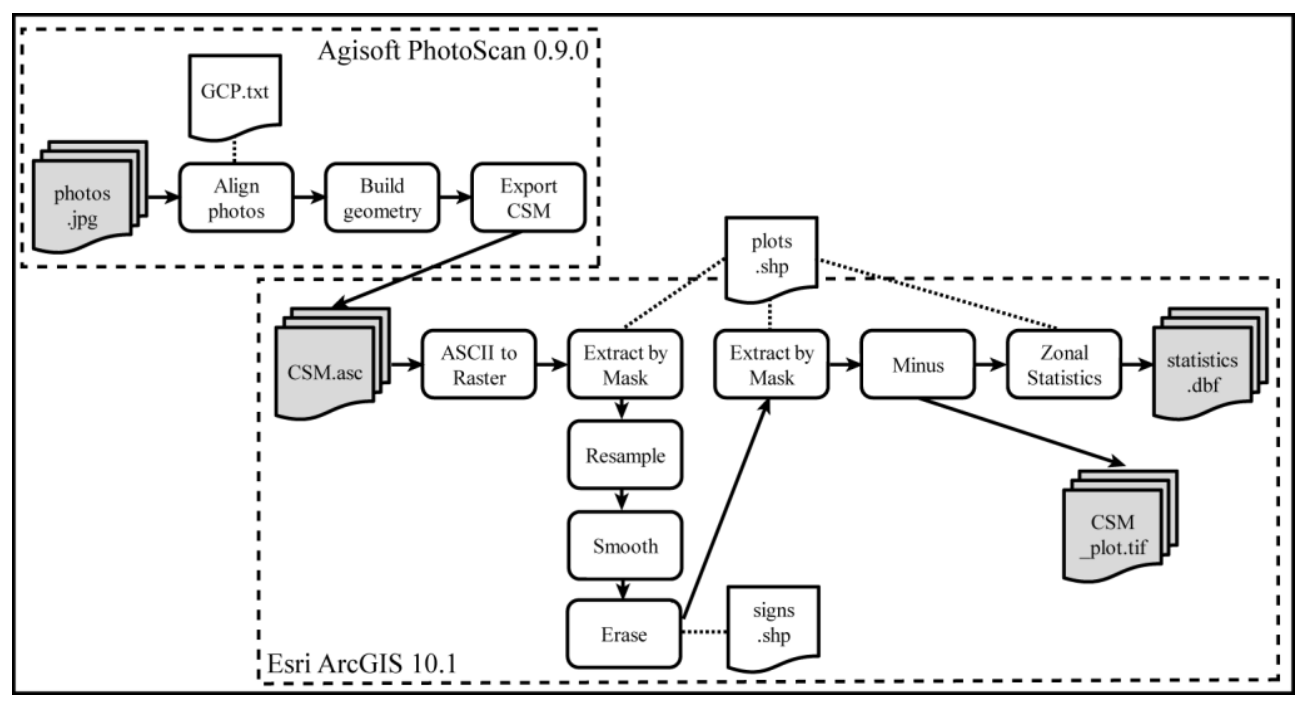

Figure 4. Data Processing workflow for generation of CSM (CSM.asc) from RGB images captured by UAV-system (photos.jpg) in Agisoft PhotoScan and further processing for analysis in Esri ArcGIS.

The flying height was set to $50 \mathrm{~m}$, resulting in three flight strips with a $44 \%$ side and $90 \%$ forward overlap (Figure 2). Each flight strip was captured in a separate flight due to the short flight time of the UAV-system because of limited battery capacity. The RGB sensor was mounted in a fixed nadir position and orientation.

The flights were carried out in mostly clear sky conditions at low wind speed ( 0 to 2 on Beaufort scale) in the early mornings between 05:30 and 07:00 am. Due to the time zone, sunrise in this area takes place around 03:00 am, resulting in high air temperatures between 25 and $35^{\circ} \mathrm{C}$ in the early morning.

\subsection{Processing}

Data processing was carried out according to the workflow presented in Figure 4. The images captured by the UAVsystem were processed into CSMs using the multi-view 3D reconstruction software Agisoft PhotoScan 0.9.0 (Agisoft, 2013). The software utilizes a structure from motion (SfM) algorithm (Verhoeven, 2011). Hence, the advantage of being able to process data despite unknown camera positions can be exploited. Three tiles were generated for each date of data acquisition according to the three flight strips. This allowed for manageable computation time and size of datasets. The CSMs have a $0.01 \mathrm{~m}$ resolution with an average point count of 12 million points.

Coordinates were assigned to the visible GCPs in the images. After that, photo alignment and model generation was carried out. An ASCII-file was exported which enabled further processing in ArcGIS. Those ASCII-files were converted to raster files which allowed for better performance. A shapefile containing the plot outlines reduced by $0.6 \mathrm{~m}$ in size served as a mask for extracting the area of interest (AOI). The reduction in size was carried out to account for plot boundary effects. Raster size was then resampled to $0.1 \mathrm{~m}$ and data was smoothed with a focal mean of $3 \times 3$ pixel rectangles. Signs with labels had been placed in the plots for indication of cultivar and treatment. These had to be erased from the models using a shapefile containing rectangles at the position of those signs. The resulting models could then be subtracted from each other resulting in two datasets containing information about growth between the three dates. In a last step, general statistics were generated on a plot size basis.

The concept of CSMs includes generation of absolute plant heights as well as growth monitoring. Due to frequently varying water levels in each of the 54 plots, no ground plane could be generated and be used as a basis for calculation of absolute heights.

Each dataset was analysed on a plot based scale. Some of the generated CSMs showed unrealistic height values and were removed from the analysis in dataset selection a (Table 1). For dataset selection $b$, a part of the field was chosen where both CSMs for growth period 1 (GP1) (04.-09.07.) and growth period 2 (GP2) (09.-17.07.) had good quality and were not affected by boundary effects.

\section{RESULTS}

\subsection{Statistics}

A first investigation of the resulting models showed that some of the datasets differed in height from the rest. For GP1 data of flight strip 1 and for GP2 data of flight strip 2 were excluded from statistical analysis in dataset selection $a$ (Table 1, 2).

\begin{tabular}{|l|cc|cc|cc|}
\hline dataset & \multicolumn{2}{|c|}{ all values } & \multicolumn{2}{c|}{ selection a } & \multicolumn{2}{c|}{ selection b } \\
\hline growth & GP1 & GP2 & GP1 & GP2 & GP1 & GP2 \\
\hline min & -0.433 & -0.514 & -0.322 & -0.284 & -0.019 & 0.078 \\
max & 0.508 & 0.786 & 0.408 & 0.474 & 0.324 & 0.474 \\
range & 0.941 & 1.300 & 0.730 & 0.758 & 0.343 & 0.396 \\
mean & 0.144 & 0.069 & 0.130 & 0.143 & 0.206 & 0.255 \\
std. & 0.040 & 0.051 & 0.037 & 0.040 & 0.026 & 0.036 \\
\hline
\end{tabular}

Table 1. Descriptive statistics of plant heights [m] derived from CSMs for all data and selected datasets $a$ and $b$ according to GP. 


\begin{tabular}{|ll|cc|cc|}
\hline & cultivar & $\begin{array}{c}\text { Kongyu } \\
131\end{array}$ & $\begin{array}{c}\text { Longjing } \\
21\end{array}$ & $\begin{array}{c}\text { Kongyu } \\
131\end{array}$ & $\begin{array}{c}\text { Longjing } \\
21\end{array}$ \\
\hline & growth & \multicolumn{2}{|c|}{ GP1 } & \multicolumn{2}{|c|}{ GP2 } \\
\hline $\mathrm{Da}$ & all values & 0.129 & 0.157 & 0.083 & 0.121 \\
tas & selection a & 0.129 & 0.131 & 0.155 & 0.129 \\
et & selection b & 0.206 & no data & 0.255 & no data \\
\hline
\end{tabular}

Table 2. Mean plant heights [m] derived from CSMs according to cultivar and GP for all data and selected datasets $a$ and $b$.

Dataset selection $b$ (Table 1,2) was chosen for closer analysis of the core part of the field, where realistic results for both growth periods could be obtained (Figure 7).

Overall plant growth (Table 1) obtained from the CSMs varies between -0.514 and $0.786 \mathrm{~m}$ for all values while selections $a$ and $b$ show lower values (a: $-0,322$ to $0.474 \mathrm{~m}$, b: -0.019 to $0.474 \mathrm{~m})$. Regarding all data, the mean plant growth declines between GP1 and GP2 from 0.144 to $0.069 \mathrm{~m}$, while for selections $a$ and $b$ growth increases slightly (a: 0.13 to $0.143 \mathrm{~m}, \mathrm{~b}: 0.206$ to $0.255 \mathrm{~m}$ ). The standard deviation varies between 0.051 and $0.026 \mathrm{~m}$. In all datasets the standard deviation is about $0.01 \mathrm{~m}$ higher for GP2 compared to GP1 e.g. 0.037 compared to 0.040 (selection a).

Table 2 shows the mean plant growth differentiated by cultivars Kongyu131 and Longying21. For all values the growth of Longjing21 $(0.157$ and $0.121 \mathrm{~m})$ is higher than for Kongyu131 (0.129 and $0.083 \mathrm{~m})$ for both GP1 and GP2. Regarding selection a, growth is nearly similar for GP1 $(0.129$ and $0.131 \mathrm{~m})$ and higher for Kongyu131 for GP2 $(0.155$ and $0.129 \mathrm{~m})$. Selection $\mathrm{b}$ only contains data for Kongyu131.

\subsection{Crop Surface Models}

An example of a CSM with a $0.01 \mathrm{~m}$ resolution is shown in Figure 5. Orange areas indicate high and green areas indicate low heights. The highest areas are in the centre of the field, while heights decrease towards the north and south. The red areas in the western part show the positions of the signs that had been placed in the field. The dark green areas in the north and in the south show the water channels used for irrigation.

\subsection{Crop Growth}

A map of plant growth of GP1 of flight strip 2 is presented in Figure 6. Values under $-0.08 \mathrm{~m}$ are coloured in grey. Values higher than that change from yellow to red, while red indicates the highest growth. Areas with the highest growth between 0.244 and $0.405 \mathrm{~m}$ are located in the centre. Growth is decreasing towards north and south where the grey areas are located. A similar trend can be seen in Figure 5.

An example of a detailed plot analysis is given in Figure 7 of the plots in centre of flight strip 3. Plant growth of GP1 (a) on the left and GP2 (b) on the right is compared. For GP1 growth is lower than for GP2. For GP1 (a) plots 182, 141 and 142 show the highest growth, while plot 171 has the lowest values. In GP2 (b) plots 131, 182, 141 and 151 show the highest values and 161 has the lowest values. Growth variability in the plots can be detected as well, e.g. the northwest corner of plot 171 growing stronger than the southeast corner.

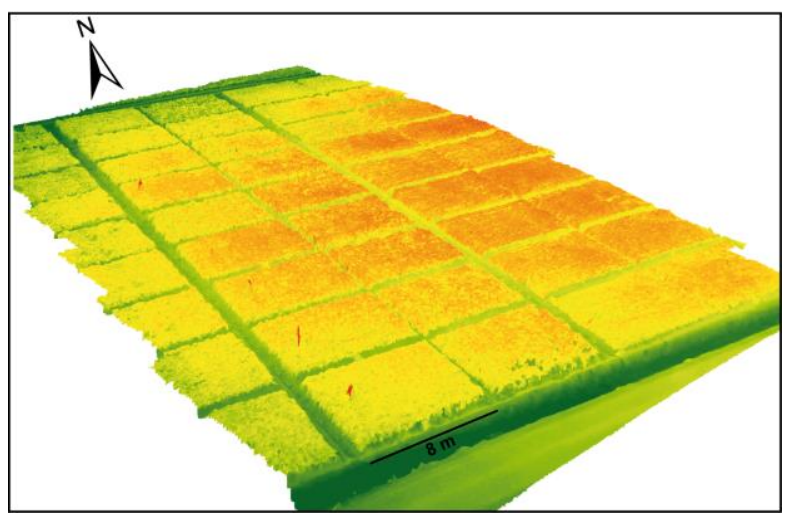

Figure 5. CSM showing flight strip 2 - orange areas indicate high and green areas indicate low heights (09.07.12)

(Esri ArcScene, height 2 times exaggerated).

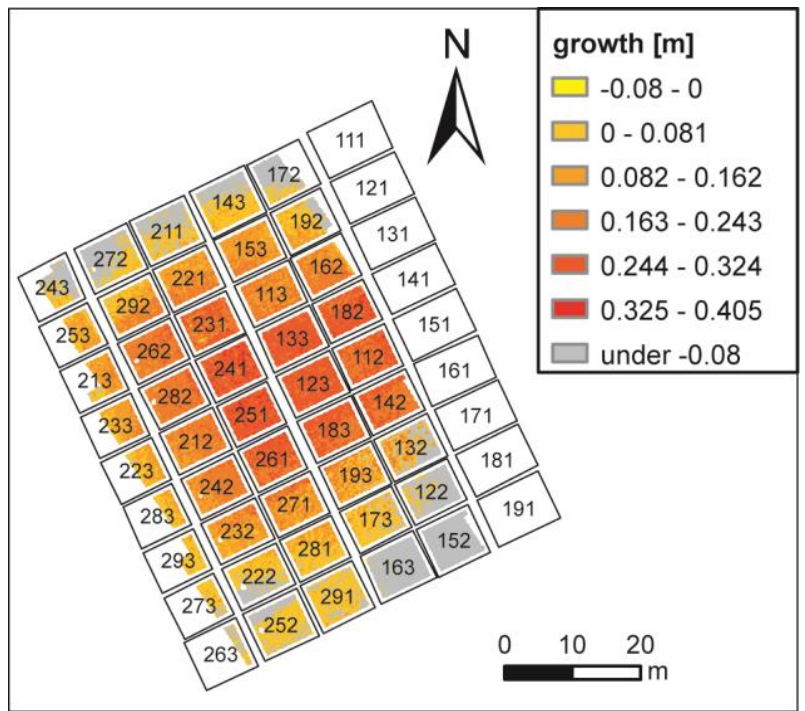

Figure 6. Plant growth of flight strip 2 for GP1 (Esri ArcMap).

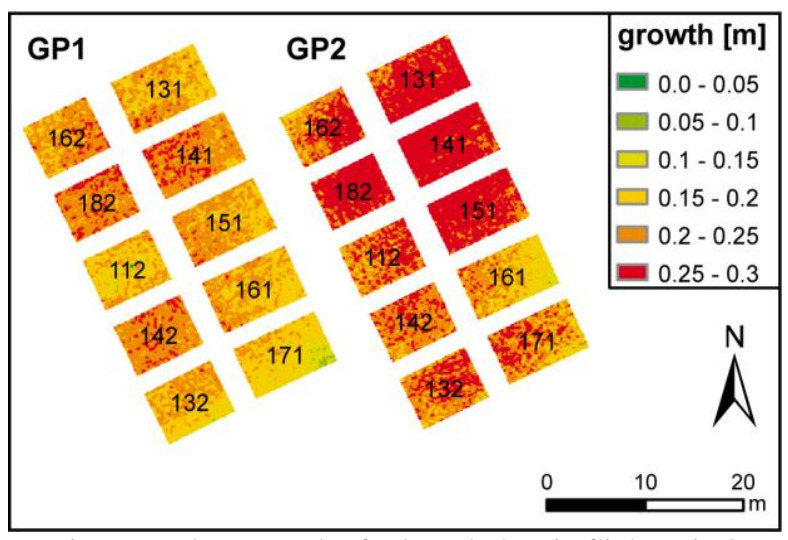

Figure 7. Plant growth of selected plots in flight strip 3 (selection $b$ ) for GP1 and GP2 (Esri ArcMap).

\section{DISCUSSION \& CONCLUSION}

Choosing a UAV for monitoring a remote small sized study area like the one presented here, enables multi-temporal data acquisition at very low cost and with high flexibility. 
Although the platform is performing well, some improvements are possible. For future campaigns, a camera holder enabling pitch and roll compensation during the flight has been mounted. A camera with a higher image resolution (Panasonic Lumix DMC GX1, 16 M pixel) is used. It is triggered electrically, which has the advantage of a more reliable image acquisition.

Visibility and distribution of the GCPs was satisfying. A disadvantage was that for measurement of the GCP positions, only a GPS with a low accuracy of $1 \mathrm{~m}$ was available. This can be regarded as the main source of error during the process of CSM generation and the results of the whole data analysis. The availability of point clouds generated from terrestrial laser scanning offers the chance of putting up a local coordinate system with a higher accuracy resulting in models of significantly higher accuracy.

The developed flight plan with $44 \%$ side and $90 \%$ forward overlap and $50 \mathrm{~m}$ flying height produced images at very high resolution with sufficient overlap for CSM generation. Comparable studies use similar flight plans with overlaps of 70 to $95 \%$ (Haala \& Rothermel, 2012; Hartmann et al., 2012). The area of image acquisition should be extended further across the borders of the field to account for errors at the CSM edges.

Model generation using Agisoft PhotoScan was comfortable and well suited for the task of handling unregistered aerial images. Comparison of other software like Bundler or Patchbased Multi-view Stereo Software Version 2 (PMVS2) already stated a good performance of Agisoft PhotoScan (Neitzel \& Klonowski, 2011).

Results of the plant growth analysis show that there is a big range of values of up to one meter (see Table 1) in the dataset, which is probably linked to the quality of the resulting CSMs; since the growth ranges of the datasets of selection $a$ and $b$ are considerably lower (Table 1). The fact that negative values of up to $-0.5 \mathrm{~m}$ occur in the data evinces the limits of data quality obtainable. Again, the occurrence of such values is probably linked to the low accuracy of the GCPs.

Absolute values obtained for the mean plant growth have a realistic magnitude between 0.06 and $0.26 \mathrm{~m}$ (Table 1) for the regarded phenological stage. This can be stated due to frequent observation of rice fields in this region since 2007 (Kang, 2013).

When comparing the cultivars, no differentiation in growth can be observed. Differences are in the magnitude of a few centimetres (e.g. 0.129 compared to 0.157). The dataset where all values had been used suggests a higher growth for Longjing21, while selection a suggests growth being higher for Kongyu131 for GP2. A reason can be that no actual difference in the growth of the two cultivars exists for the regarded GP (about 1 month) or the quality of the CSMs was not sufficient for such sort of analysis.

When analysing the spatial distribution of growth (Figures 5,6) it can be clearly seen that plant heights are decreasing towards the northern and southern ends of the fields. This could be due to inhomogeneity in the field e.g. soil quality or other factors influencing plant growth. Since
Figure 6 gives the impression of a radial decrease of growth towards the edges, another reason seems more likely: It could be a barrel effect in the CSMs which is resulting in lower heights at the edges of the CSM. This problem can be addressed by extending the area of interest during the flights and by solving the problem of inaccurate registration of the GCPs.

In the centre of the field where this effect is not masking the results, the variability of growth in the plots can be shown with very high detail (Figure 7). This proves that the method is suitable for detection of small scale variability (here: $0.1 \mathrm{~m}$ raster resolution) in plant growth.

Due to the strong barrel effect influencing the models, no analysis of the different treatments of $n$-fertilizer (Figure 2) was performed. This step can be added to the analysis as well as comparison of the derived CSMs with the additional data e.g. models from terrestrial laser scanning or hyperspectral reflection data.

In summary it can be stated that the method of multitemporal CSM generation from UAV-based data, is applicable to rice. For the reliable modeling of absolute plant heights and a differentiation of cultivars and treatment the model quality needs to be improved, which is possible through improved data processing and will be done in the future.

\section{ACKNOWLEDGEMENTS}

The authors acknowledge the funding of the CROP.SENSe.net project in the context of Ziel 2-Programms NRW 2007-2013 "Regionale Wettbewerbsfähigkeit und Beschäftigung (EFRE)" by the Ministry for Innovation, Science and Research (MIWF) of the state North Rhine Westphalia (NRW) and European Union Funds for regional development (EFRE) (005-1103-0018) while the preparation of the manuscript.

\section{REFERENCES}

Agisoft, 2013: http://www.agisoft.ru (29. Apr. 2013).

Bendig, J., Bolten, A. \& Bareth, G., 2012: Introducing a lowcost Mini-UAV for thermal- and multispectral-imaging. International Archives of the Photogrammetry, Remote Sensing and Spatial Information Sciences, XXXIX(B1), pp. 345-349.

Bendig, J., Bolten, A., Bareth, G., 2013: UAV-based imaging for multi-temporal, very high resolution crop surface models (CSMs) to monitor crop growth variability. Photogrammterie, Fernerkundung, Geoinformation, in review.

Berni, J. A. J., Zarco-Tejada, P. J., Suárez, L., Fereres, E., 2009: Thermal and narrowband multispectral remote sensing for vegetation monitoring from an unmanned aerial vehicle. IEEE Transactions on Geoscience and Remote Sensing, 47(3), pp. 722-738.

Haala, N., Rothermel, M., 2012: Dense multi-stereo matching for high quality digital elevation models. Photogrammetrie, Fernerkundung, Geoinformation, 4, pp. 331-343.

Hansen, P. M., Schjoerring, J. K., 2003: Reflectance measurement of canopy biomass and nitrogen status in wheat 
crops using normalized difference vegetation indices and partial least squares regression. Remote Sensing of Environment, 86(4), pp. 542-553.

Hardin, P. J., Jensen, R. R., 2011: Introduction - Small-scale unmanned aerial systems for environmental remote sensing. GIScience \& Remote Sensing, 48(1), pp. 1-3.

Hartmann, W., Tilch, S., Eisenbeiss, H., Schindler, K, 2012: Determination of the UAV position by automatic processing of thermal images. International Archives of the Photogrammetry, Remote Sensing and Spatial Information Sciences, XXXIX(B6), pp. 111-116.

Hendrickx, M., Gheyle, W., Bonne, J., Bourgeois, J., De Wulf, A., Goossens, R., 2011: The use of stereoscopic images taken from a microdrone for the documentation of heritage - An example from the Tuekta burial mounds in the Russian Altay. Journal of Archaeological Science, 38, pp. 2968-2978.

Hisystems GmbH, 2013: http://www.hisystems.de (29. Apr. 2013).

Hoffmeister, D., $\quad$ Bolten, A., $\quad$ Curdt, C., Waldhoff, G., Bareth, G., 2010: High resolution Crop Surface Models (CSM) and Crop Volume Models (CVM) on field level by terrestrial laserscanning, SPIE Proceedings, 7840.

Kang, Y., Li, F. Gnyp, M. L., Miao, Y., Bareth, G., Chen, X., 2013: Remotely detecting canopy nitrogen concentration and uptake of paddy rice in the Northeast China Plain. ISPRS Journal of Photogrammetry and Remote Sensing, 78(2013), pp. 102-115.

Miao, Y., Stewart, B. A., Zhang, F., 2012: Long-term experiments for sustainable nutrient management in China. A review. Agronomy for Sustainable Development, 31, pp. 397414.
Martinsanz, G. P., 2012: Special issue "Unmanned Aerial Vehicles (UAVs) based remote sensing". https://www.mdpi.com/journal/remotesensing/special_issues/ uav (29. Apr. 2013).

Mulla, D. J., 2012: Twenty five years of remote sensing in precision agriculture: Key advances and remaining knowledge gaps. Biosystems Engineering, XXX, pp. 1-14.

Neitzel, F., Klonowski, J., 2011: Mobile 3D mapping with a low-cost UAV system. International Archives of the Photogrammetry, Remote Sensing and Spatial Information Sciences, XXXVIII-1(C22), pp. 1-6.

Panasonic, 2013: http://94.23.55.209/index.php/Cameras/Ca mera-Sensor-Database/Panasonic/Lumix-DMC-GF3 Apr. 2013)

Peng, S., Buresh, R. J., Huang, J., Yang, J., Zou, Y., Zhong, X., Wang, G., Zhang, F., 2006: Strategies for overcoming low agronomic nitrogen use efficiency in irrigated rice systems in China. Field Crops Research, 96, pp. 37-47.

Verhoeven, G., 2011: Taking computer vision aloft archaeological three-dimensional reconstructions from aerial photographs with PhotoScan. Archaeolical Prospection, 18, pp. 67-73.

Watts, A. C., Ambrosia, V. G., Hinkley, E. A., 2012. Unmanned aircraft systems in remote sensing and scientific research: Classification and considerations of use. Remote Sensing, 4, pp. 1671-1692.

Thenkabail, P. S., Smith, R. B., Pauw, E. D., 2000: Hyperspectral vegetation indices and their relationship with agricultural crop characteristics. Remote Sensing of Environment, 71(2), pp. 152-182. 